Article

\title{
Folate Functionalized PLGA Nanoparticles Loaded with Plasmid pVAX1-NH36: Mathematical Analysis of Release
}

\author{
Cindy Alejandra Gutiérrez-Valenzuela ${ }^{1}$, Patricia Guerrero-Germán ${ }^{2}$, Armando Tejeda-Mansir ${ }^{3}$, \\ Reynaldo Esquivel ${ }^{1}$, Roberto Guzmán-Z ${ }^{4}$ and Armando Lucero-Acuña ${ }^{2, *}$ \\ 1 Nanotechnology Graduate Program, Department of Physics, University of Sonora, \\ Hermosillo 83000, Mexico; cindya.gutierrez@yahoo.com (C.A.G.-V.); resquivelgo@conacyt.mx (R.E.) \\ 2 Department of Chemical and Metallurgical Engineering, University of Sonora, Hermosillo 83000, Mexico; \\ pguerrero@iq.uson.mx \\ 3 Department of Scientific and Technological Research, University of Sonora, Hermosillo 83000, Mexico; \\ atejeda@guayacan.uson.mx \\ 4 Department of Chemical and Environmental Engineering, University of Arizona, Tucson, AZ 85721, USA; \\ guzmanr@email.arizona.edu \\ * Correspondence: armando.lucero@unison.mx; Tel.: +52-662-259-2105
}

Academic Editor: Jordi Puiggalí

Received: 15 October 2016; Accepted: 11 November 2016; Published: 25 November 2016

\begin{abstract}
Plasmid DNA (pVAX1-NH36) was encapsulated in nanoparticles of poly-DL-lactic-coglycolic (PLGA) functionalized with polyethylene glycol (PEG) and folic acid (PLGA-PEG-FA) without losing integrity. PLGA-PEG-FA nanoparticles loaded with pVAX1-NH36 (pDNA-NPs) were prepared by using a double emulsification-solvent evaporation technique. PLGA-PEG-FA synthesis was verified by FT-IR and spectrophotometry methods. pVAX1-NH36 was replicated in Escherichia coli (E. coli) cell cultures. Atomic force microscopy (AFM) analysis confirmed pDNA-NPs size with an average diameter of 177-229 nm, depending on pVAX1-NH36 loading and zeta potentials were below $-24 \mathrm{mV}$ for all preparations. In vitro release studies confirmed a multiphase release profile for the duration of more than 30-days. Plasmid release kinetics were analyzed with a release model that considered simultaneous contributions of initial burst and degradation-relaxation of nanoparticles. Fitting of release model against experimental data presented excellent correlation. This mathematical analysis presents a novel approach to describe and predict the release of plasmid DNA from biodegradable nanoparticles.
\end{abstract}

Keywords: drug delivery; pVAX1-NH36; mathematical analysis; copolymer synthesis; leishmaniasis; folate nanoparticles

\section{Introduction}

Research related to the use of nano-systems in drugs and genes has increased in recent decades due to a number of innovative applications of nano-systems in health-related issues. The global market for drug delivery systems and genes is projected to reach $\$ 175.6$ billion by 2016 , which represents an increase of $\$ 44$ billion from 2010 [1]. The main focus of such nano-systems administered drugs and genes research is to deliver a specific amount of the component of interest in the right place, at the right time, and nano-formulation has received the most attention out of others. The exposure of cells/tissues/tumors to therapeutic agents increases when nano-formulations are designed as drug delivery vehicles. Various therapeutic and immunization agents, such as anticancer drugs and plasmid DNA (pDNA), could be encapsulated and administered simultaneously to enhance the effectiveness of such therapy and/or immunization [2,3]. 
Among different materials used for drug delivery, one of the most successful is poly (ester): poly-DL-lactic-co-glycolic (PLGA), which has immense potential as a drug delivery vehicle as well as a platform for tissue engineering [4,5]. PLGA is a biodegradable polymer mostly used for developing nano-medicines. It hydrolyzed in body to produce lactic acid and glycolic acid monomers, which subsequently degraded to carbon dioxide and water $[4,6,7]$. Degradation rates and structural/mechanical properties of PLGA depend on the proportion of monomers formed $[4,6]$. PLGA contains carboxylic acid, which can also be functionalized with other molecules before or after the preparation of nanoparticles (NPs). One of the possible modifications of PLGA is coupling with polymer polyethylene glycol (PEG). It has been shown that PEG-coated carriers have increased circulation half-life in blood, reduced rate of uptake by the liver and prevented its recognition by the mononuclear phagocytic system [8]. In addition, they can facilitate a full control of drug release rates [9]. Furthermore, PLGA and PEG are already approved by the Food and Drug Administration (FDA) and the European Medicine Agency (EMA) and used in therapeutic devices [4,10].

Another desirable characteristic of these nano-systems is possible functionalization to target specific cells. Selective gene delivery to folate receptor overexpressing tissues by decorating NPs with folic acid (FA) as targeting ligand is of great interest [11-13]. Chemical coupling of PLGA, PEG and FA will enable us to produce NPs with blood compatibility, drug release rate control and targeting characteristics. Polymer NPs have been prepared using different methods and the most common of them is emulsification-solvent evaporation technique $[4,6,14,15]$. Other available methods are emulsification-diffusion of solvent $[16,17]$, nanoprecipitation $[18,19]$ and others.

Leishmaniasis is the third most important vector-borne disease worldwide, caused by protozoan Leishmania parasites that are transmitted by the bite of infected sandflies. There is consensus that vaccines ought to become a major tool in the control of such group of diseases [20]. In the specific case of leishmaniasis, a recent review confirms that, to date, there is no drug therapy or human vaccine available [21]. The inherent features of pDNA make them promising vaccine candidates in a variety of diseases like leishmaniasis [22-25] and pDNA-based therapeutic and immunization methods relay on genes transfer to human cells to encode the synthesis of a protein, which, when expressed, carries an immunizing or therapeutic action. In this regard, the NH36 gene is one of the candidates for DNA vaccines against leishmaniasis and has already been tested in several animal models [26,27]. On the other hand, reports have shown limitations and restrictions in transport of pDNA through intramuscular, cutaneous and intracutaneous routes [28]. Therefore, an improved system for transferring pDNA to a specific site are necessary to address these limitations. Wang et al. [28] tried to solve this problem by encapsulating pDNA into micro-particles of biodegradable polymers. In this way, they protect the plasmid from being digested by ribonucleases and also assist the transport and control release rate. Recent reports on pDNA encapsulation into polymeric NPs are focused on cancer gene therapy and vaccination applications [29-33] where the authors have addressed encapsulation of pDNA into polymeric NPs by using different approaches. In general, the process of pDNA encapsulation into polymeric NPs involves several steps and preparation parameters that should be modulated according to desired NPs characteristics. Experimental pDNA release from biodegradable matrices have been reported for different plasmids [34-36] and, to the best of our knowledge, only few efforts to mathematically describe the pDNA release kinetics have been reported, where the entire plasmid release process has been attributed to the diffusion mechanism [37]. Contrary to pDNA release kinetics, mathematical analysis of drug release from biodegradable matrices has been extensively reported in literature by considering one or more mechanisms of release $[38,39]$.

In this present research, a preparation process of biodegradable nanoparticles loaded with plasmid pVAX1-NH36 was developed. pVAX1-NH36 was replicated using E. coli bacteria and purified to obtain a pure plasmid, followed by capsuling into polymeric NPs prepared with PLGA-PEG-FA by using different theoretical DNA loadings. Several techniques were used to characterize NPs such as dynamic light scattering, laser doppler electrophoresis, atomic force microscopy (AFM), high performance liquid chromatography-hydrophobic interaction chromatography (HPLC-HIC) and agarose electrophoresis. 
pVAX1-NH36 release was evaluated in vitro under physiological conditions and release kinetics were analyzed by using an effective mathematical release model that simultaneously considers the mechanism of initial burst as well as mechanism of degradation-relaxation of NPs [40]. Mechanism of initial burst was calculated using first-order rate burst release and the mechanism of release due to bulk degradation of polymer or NPs relaxation was evaluated in an analogous way to thermal decomposition of potassium permanganate crystals [41,42]. These two mechanisms were linearly coupled to effectively describe the entire plasmid release process.

\section{Materials and Methods}

\subsection{Materials}

PLGA acid terminated (50/50 DL-lactide/glycolide copolymer, IV midpoint $0.2 \mathrm{dL} / \mathrm{g}$ ) was obtained from Corbion Purac, Gorinchem, The Netherlands. Glycerol, yeast extract, tryptone, potassium sulfate dibasic and monobasic, kanamycin, $\mathrm{N}$-Hydroxysuccinimide (NHS), dimethyl sulfoxide (DMSO), methanol and diethyl ether were obtained from Sigma-Aldrich, Inc., Milwaukee, WI, USA. Supercoiled DNA ladder was obtained from Invitrogen, Corp., Carlsbad, CA, USA. $O, O^{\prime}$-Bis(2-aminopropyl) polypropylene glycol-block-polyethylene glycol-block-polypropylene glycol (PEG) of 1900 amu was obtained from Fluka, Buchs, Switzerland. Folic acid (FA) and polyvinyl alcohol (Mowiol ${ }^{\circledR} 4-88 \mathrm{Mw} \sim 31,000 \mathrm{amu}, \mathrm{PVA}$ ) was obtained from Sigma Aldrich, Inc., St. Louis, MO, USA. N,N'-Dicyclohexylcarbodiimide (DCC) was obtained from Alfa Aesar, Ward Hill, MA, USA. Dichloromethane (DCM) was obtained from Fisher Scientific Inc., Fair Lawn, NJ, USA.

\subsection{Cell Culture and $p V A X 1-N H 36$ Recovery}

Plasmid pVAX1-NH36 was produced by cultivation of recombinant Escherichia coli DH5 $\alpha$. Cell package was supplied by the Department of Scientific and Technological Research at the University of Sonora, Hermosillo, Sonora, Mexico. The size of the plasmid was $4.0 \mathrm{kbp}$ and contains an antibiotic resistance gene against kanamycin for selection purposes. Host strain was maintained in $30 \%(v / v)$ glycerol at $-80{ }^{\circ} \mathrm{C}$. Culture medium consisted of a terrific broth supplemented with glycerol and kanamycin with the following composition: $13.0 \mathrm{~g} / \mathrm{L}$ glycerol, $24.0 \mathrm{~g} / \mathrm{L}$ yeast extract, $12.0 \mathrm{~g} / \mathrm{L}$ tryptone, $12.54 \mathrm{~g} / \mathrm{L}$ potassium phosphate dibasic, $2.31 \mathrm{~g} / \mathrm{L}$ potassium phosphate monobasic and $50.0 \mu \mathrm{g} / \mathrm{mL}$ kanamycin. Cultures were started from glycerol stocks inoculated into medium and grown in shake flasks at $37^{\circ} \mathrm{C}$ on an orbital shaker at $250 \mathrm{rpm}$ for eight hours. Cell culture concentration was obtained by using an equipment Perkin Elmer UV/VIS spectrometer lambda 2S (Perkin-Elmer de Mexico, Monterrey, Nuevo Leon, Mexico) measuring optical density (OD) at $600 \mathrm{~nm}$. After fermentation, culture was centrifuged and pellet was used for plasmid recovery. pVAX1-NH36 was purified using GenElute ${ }^{\mathrm{TM}}$ plasmid Maxiprep Kit (Sigma Aldrich Co., St. Louis, MO, USA) according to manufacturer's instructions. Plasmid solution was concentrated using a stirred ultrafiltration cell system Millipore 8050 (Millipore Corporation, Bedford, MA, USA) with regenerated cellulose ultrafiltration membrane with MWCO of 100,000 Da (Millipore Corporation, Billerica, MA, USA). Quantification of pVAX1-NH36 was carried out by measuring OD at $260 \mathrm{~nm}$ in a NanoDrop 1000 spectrophotometer (Thermo Fisher Scientific Inc., Waltham, MA, USA).

\subsection{Polymer Modification}

Synthesis of PLGA-PEG-FA was carried out in two steps: PLGA activation and coupling of PEG, followed by FA conjugation [43,44], as shown schematically in Figure 1. Briefly, PLGA was activated by DCC and NHS in DCM with a molar ratio of PLGA:DCC:NHS = 1:5:5, where reaction was carried out overnight at room temperature under nitrogen atmosphere. The resultant solution was filtered (Watman \#1 filter) to remove dicyclohexylurea. For PEG coupling, activated PLGA solution was treated with PEG at a molar ratio of 1:5 and left to react overnight under nitrogen atmosphere. Polymer was washed by three cycles of precipitation using three volumes of cold organic mixture of methanol-diethyl 
ether (50/50) followed by centrifugation, discarding supernatant and resuspending in DCM. In the last cycle, after centrifugation and removal of supernatant, polymer was dried under vacuum. To attach FA to polymer, PLGA-PEG was dissolved in DMSO and treated with FA in DMSO at a molar ratio of 1:5 with overnight stirring at room temperature under nitrogen atmosphere. Then, the solution was filtrated (Watman \#1 filter) and set to dialyze in a Spectra/Por ${ }^{\circledR}$ Membrane MWCO 3500 in $5 \mathrm{mM}$ sodium carbonate ( $\mathrm{pH}$ 8.0) buffer for four hours. Next, buffer was replaced and sample was dialyzed for four more hours, followed by overnight dialysis in deionized water. Finally, PLGA-PEG-FA was lyophilized to remove water and trace amounts of organic solvent in lyophilizer Freezone 4.5 (Labconco, Kansas City, MO, USA). The structure of the copolymer was confirmed by Fourier transform infrared spectroscopy in the equipment FT-IR spectrometer spectrum (Perkin Elmer, Llantrisant, UK). For FT-IR measurements, polymer was set directly onto an attenuated total reflectance (ATR) crystal and spectrum was collected in a range between 4000 and $550 \mathrm{~cm}^{-1}$ with a resolution of $4 \mathrm{~cm}^{-1}$ (16 scans per sample).

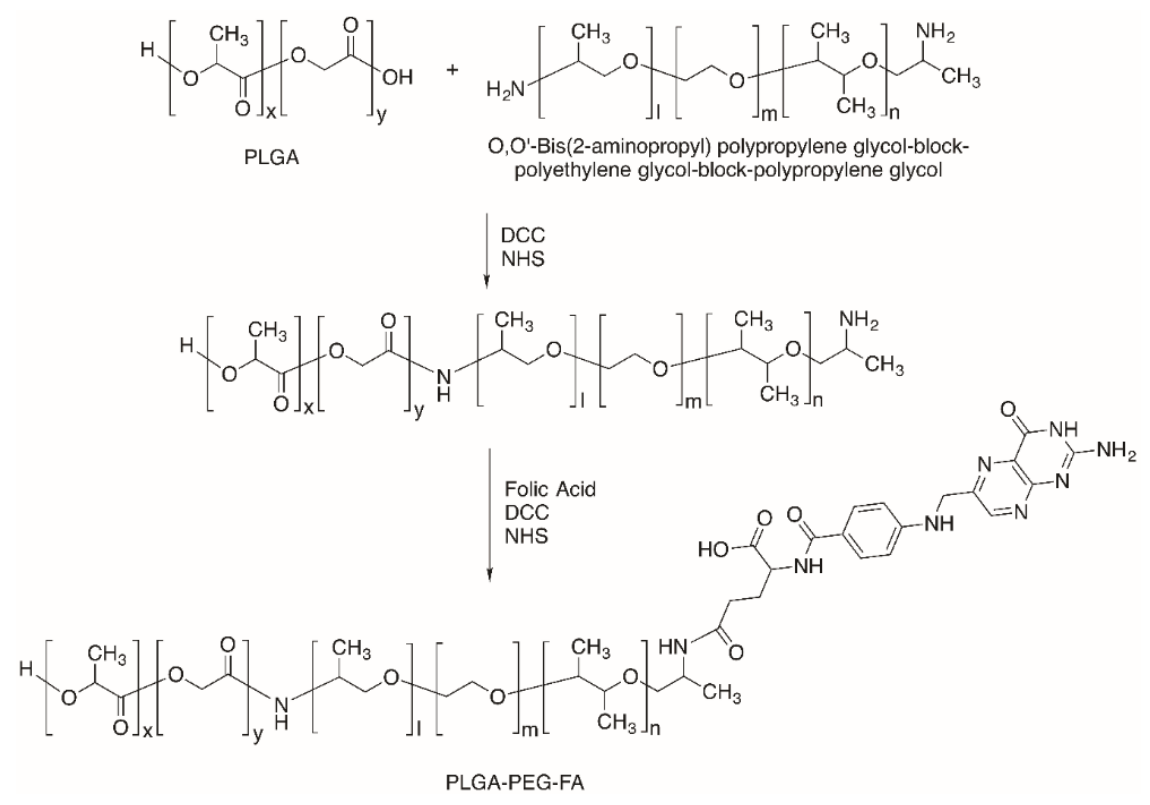

Figure 1. Schematic of the synthesis of the polymer poly-DL-lactic-co-glycolic (PLGA) polyethylene glycol (PEG) folic acid (PLGA-PEG-FA). First, PLGA carboxylic acid is activated and coupled to a primary amine of PEG (PLGA-PEG). Then, a carboxylic acid from FA is activated and conjugated to the primary amine of PLGA-PEG to finally obtain PLGA-PEG-FA.

\subsection{Preparation of $p D N A-N P S$}

Folic acid functionalized PLGA NPs loaded with pVAX1-NH36 (pDNA-NPs) were prepared by using a double emulsification technique followed by solvent evaporation [4,45-47]. Three different theoretical pVAX1-NH36 loadings (TDL) - ratio of initial amount of pVAX1-NH36 used in the process with respect to initial amount of polymers-were used in the preparations. Briefly, a mixture with $1 \mathrm{~mL}$ of pVAX1-NH36 in aqueous phase $(0.2 \%, 0.5 \%$ and $0.8 \%$ TDL) and polymers were dissolved in $4 \mathrm{~mL}$ of DCM (25 mg of PLGA and $25 \mathrm{mg}$ of PLGA-PEG-FA) and emulsified using an ice bath with a QSonica 500 sonicator (QSonica LLC, Newtown, CT, USA) operating at $26.5 \mu \mathrm{m}$ of amplitude for $1 \mathrm{~min}$. Then, $8 \mathrm{~mL}$ of $3 \%$ PVA were added and a second emulsification at $90 \mu \mathrm{m}$ of amplitude in an ice bath was performed for $1 \mathrm{~min}$. Organic solvent was then evaporated under magnetic stirring at room temperature overnight. Next, a 2-min centrifugation cycle at $2000 \mathrm{rpm}(376 \times g)$ using a Sigma 3-30KS centrifuge (Sigma Laborzentrifugen GmbH, Osterode am Harz, Germany) was employed and supernatant was collected. Supernatant was washed by three centrifugation cycles at 19,000 rpm $(33,902 \times g)$ for $10 \mathrm{~min}$, discarding supernatant and resuspending pellet pDNA-NPs in $8 \mathrm{~mL}$ of deionized 
water. On the final centrifugation cycle, pDNA-NPs were resuspended in $4 \mathrm{~mL}$ of deionized water and freeze-dried in lyophilizer freezone 4.5 (Labconco, Kansas City, MO, USA). All experiments were in triplicate.

\subsection{Nanoparticle Characterization}

Nanoparticle size distribution and zeta potentials were measured using a zetasizer Nano ZS equipment (Malvern Instruments Ltd., Worcestershire, UK). Measurements of pDNA-NPs sizes were performed by dynamic light scattering. Each sample was measured three times with 10 runs respectively. Additionally, each sample for zeta potential was measured by duplication with at least 10 runs at constant temperature $\left(25^{\circ} \mathrm{C}\right)$ by laser doppler electrophoresis. Z-averages and zeta potentials were obtained from three independent experiments.

Surface morphology of pDNA-NPs was analyzed by scanning electron microscopy (SEM) through a field emission scanning electron microscope (JSM-7800F, JEOL, Pleasanton, CA, USA). Nanoparticle samples were prepared using formvar carbon film and air-dried for several minutes. Surface morphology was confirmed by atomic force microscopy (AFM JSP-4210, JEOL, Tokyo, Japan) in noncontact mode by using an NSC15 silicon cantilever (MikroMasch, Santa Clara, CA, USA). For surface analysis, a drop of pDNA-NPs solution was deposited onto freshly cleaved mica and air-dried for several minutes. AFM images were analyzed with WSxM software [48].

pVAX1-NH36 loading in pDNA-NPs (DL) was determined by subtracting the amount of pVAX1-NH36 recovered in wash solutions from the initial amount of pDNA added $[35,49,50]$. Free pVAX1-NH36 concentration in all solutions was measured by HPLC-HIC [51] with a source 15PHE (Phenil-Polyestirene) column of $0.46 \mathrm{~cm}$ of diameter per $10 \mathrm{~cm}$ of length on an Akta purifier 10 UPC (GE Healthcare, Chicago, IL, USA). Briefly, the column was equilibrated with $1.5 \mathrm{M}$ ammonium sulphate in Tris- $\mathrm{HCl} 10 \mathrm{mM}$ with $\mathrm{pH}$ of 8.0 at a flow rate of $1 \mathrm{~mL} / \mathrm{min}$ and a sample $(30 \mu \mathrm{L})$ was injected at the same flow rate. The column was eluted for $0.8 \mathrm{~min}$ with the same buffer used for equilibration and an isocratic elution was performed using Tris hydrochloride buffer (Tris-HCl) for $0.7 \mathrm{~min}$. After this period, ammonium sulphate concentration was increased to its initial value. This condition was maintained during the next $5.5 \mathrm{~min}$ to re-equilibrate column. Absorbance at $260 \mathrm{~nm}$ and conductivity of eluate were recorded continuously. Plasmid amount was quantified using a calibration curve obtained with a standard model plasmid prepared in a $0-60 \mu \mathrm{g} / \mathrm{mL}$ concentration range. Encapsulation efficiency (EE) was defined as a percentage of pVAX1-NH36 encapsulated in pDNA-NPs with respect to the initially added amount of pVAX1-NH36.

\subsection{In Vitro Release Study}

To evaluate in vitro pVAX1-NH36 release from pDNA-NPs, an ultracentrifugation method was used [52]. Initially, $10 \mathrm{mg}$ of freeze-dried pDNA-NPs were resuspended in $1 \mathrm{~mL}$ of phosphate buffered saline (PBS) at $\mathrm{pH} 7.4$ and incubated at $37^{\circ} \mathrm{C}$. Samples were taken periodically and centrifuged using a centrifuge (Thermo Sisher Scientific, Asheville, NC, USA) at 19,000 rpm $(32,186 \times g)$ for $10 \mathrm{~min}$ at $37^{\circ} \mathrm{C}$ to obtain pellet pDNA-NPs. The supernatants were removed and replaced with fresh buffer and pDNA-NPs were resuspended by vortex. The supernatant solution was analyzed for pVAX1-NH36 by HPLC-HIC, as described above.

\subsection{Mathematical Analysis of pDNA Release}

Release analysis of active components from biodegradable systems is a combination of a number of factors. These factors determine the contribution of mechanisms of release involved, such as initial burst, NPs degradation-relaxation and diffusion. Fredenberg et al. reported a comprehensive review that considers several physical factors, such as temperature and $\mathrm{pH}$ of the system, size and shape of NPs, drug solubility, and others [38]. The pDNA release analysis from biodegradable NPs usually considers those factors, resulting in more than one mechanism of release to describe the entire release process. One of the most relevant mechanisms of release is initial burst, which is attributed to a process 
of interfacial diffusion between solid sphere surface and liquid media. By considering that initially all pVAX1-NH36 is incorporated in NPs, kinetics of initial burst follow an exponential relationship as reported in literature [53,54].

$$
\frac{M_{t}}{M_{\infty}}=1-\exp \left(-k_{b} t\right)
$$

where $M_{t}$ is the cumulative amount of pVAX1-NH36 released at time $t, M_{\infty}$ is the cumulative amount of pDNA released at infinite time and $k_{b}$ is the initial burst constant, incorporating factors such as concentration of pVAX1-NH36 on NPs surface, surface area, interphase properties, solubility of pVAX1-NH36 and electrostatic interactions between pDNA and carrier [40].

Other mechanisms associate the amount of pDNA released with polymeric matrix degradation, which depends on factors such as $\mathrm{pH}$ of the media, incubation temperature, and larger particles degrading at faster rates than smaller ones [55]. Degradation of PLGA in aqueous solutions was carried out by hydrolysis, resulting in relaxation of NPs as evident in literature [56]. PLGA NPs degradation-relaxation effects have been explained by using the Prout-Tompkins equation $[40,41,54,55]$. This equation can also be used to describe pDNA release and could be rewritten in the following form:

$$
\frac{M_{t}}{M_{\infty}}=\frac{\exp \left[k_{r}\left(t-t_{\max }\right)\right]}{1+\exp \left[k_{r}\left(t-t_{\max }\right)\right]}
$$

where $k_{r}$ is the rate of degradation-relaxation constant and $t_{\max }$ is the time to achieve a maximum rate of pDNA release or the time to achieve $50 \%$ of release.

Hence, a linear combination of Equations (1) and (2), that simultaneously consider the stages of initial burst and NPs degradation-relaxation, was used to describe the entire pDNA release process from biodegradable NPs according to Equation (3):

$$
\begin{aligned}
\frac{M_{t}}{M_{\infty}}= & \theta_{b}\left\{1-\exp \left(-k_{b} t\right)\right\} \\
& +\left(1-\theta_{b}\right)\left\{\frac{\exp \left[k_{r}\left(t-t_{\max }\right)\right]}{1+\exp \left[k_{r}\left(t-t_{\max }\right)\right]}\right\}
\end{aligned}
$$

where the initial burst contribution fraction over the entire pDNA release process is $\theta_{b}$. Equation (3) contains four unknown parameters that were determined by adjusting the equation to experimental data of pVAX1-NH36 release by using nonlinear least-squares algorithm in MATLAB ${ }^{\circledR}$ (R2010b, MathWorks, Natick, MA, USA).

\subsection{Purity and Integrity of Plasmid}

Electrophoresis was used to determine the purity of plasmid with the purpose of verifying quality of supercoiled pVAX1-NH36. Agarose gels (1\%) were prepared in Tris-Acetic acid-Ethylenediaminetetraacetic acid (TAE) buffer $(40 \mathrm{mM}$ Tris, $20 \mathrm{mM}$ acetic acid, $1 \mathrm{mM}$ ethylenediaminetetraacetic acid, $\mathrm{pH}$ 8.0). Gels were run at $80 \mathrm{~V}(40 \mathrm{~A})$ for $100 \mathrm{~min}$ on a PowerPac ${ }^{\mathrm{TM}}$ power supply unit (Bio-Rad Laboratories, Inc., Hercules, CA, USA). DNA in gels was visualized by staining in ethidium bromide (EtBr, Sigma $0.5 \mu \mathrm{g} / \mathrm{mL}$ ) for $30 \mathrm{~min}$. Stained gels were scanned using a UVP MultiDoc-It digital imaging systems.

\section{Results and Discussion}

\subsection{Cell Culture and $p D N A$ Recovery}

Cell culture was inoculated from a previous culture at exponential growth phase. A cell culture OD of 5.47 was reached after eight hours of culture. pVAX1-NH36 recovery and purification was done according to kit protocol. pVAX1-NH36 was concentrated in the ultrafiltration system described above to obtain a plasmid stock solution with a concentration of $774.6 \mathrm{mg} / \mathrm{L}$. A ratio of A260/A280 $=1.9$ was obtained. This measurement is used to assess the purity of DNA. A ratio of $\sim 1.8$ is generally accepted 
as "pure" for DNA. If the ratio is appreciably lower, it may indicate the presence of protein, phenol or other contaminants that absorb strongly at or near $280 \mathrm{~nm}$.

\subsection{Polymer Modification}

PLGA-PEG-FA was successfully synthesized in two conjugation steps. FT-IR studies were carried out to confirm the presence of amide linkage in polymer (Figure 2). Characteristic bands for folic acid at 1452 and $1608 \mathrm{~cm}^{-1}$ are presented in functionalized copolymer and could be due to stretching vibrations of $\mathrm{C}=\mathrm{C}$ in the backbone of aromatic ring present in folic acid $[57,58]$. The most significant FT-IR absorption peak in PLGA-PEG-FA polymer was caused by the presence of -CONH- linkage. The carbonyl $(\mathrm{C}=\mathrm{O})$ and amine $(\mathrm{N}-\mathrm{H})$ groups present in amide linkage exhibited bands at 1623 and $1571 \mathrm{~cm}^{-1}$ respectively $[45,58]$. Previous works with similar polymer modifications reported characteristic FT-IR absorption peaks obtained in the present work. For example, Choy et al. [57] hybridized folic acid with layered double hydroxide by ion-exchange reaction. In the same way, Yang et al. [58] reported FA-chitosan conjugates and Boddu et al. [45] synthesized PLGA-PEG-FA. Boddu et al. work on FT-IR spectrum results matches the spectrum obtained with copolymer prepared in the present work.

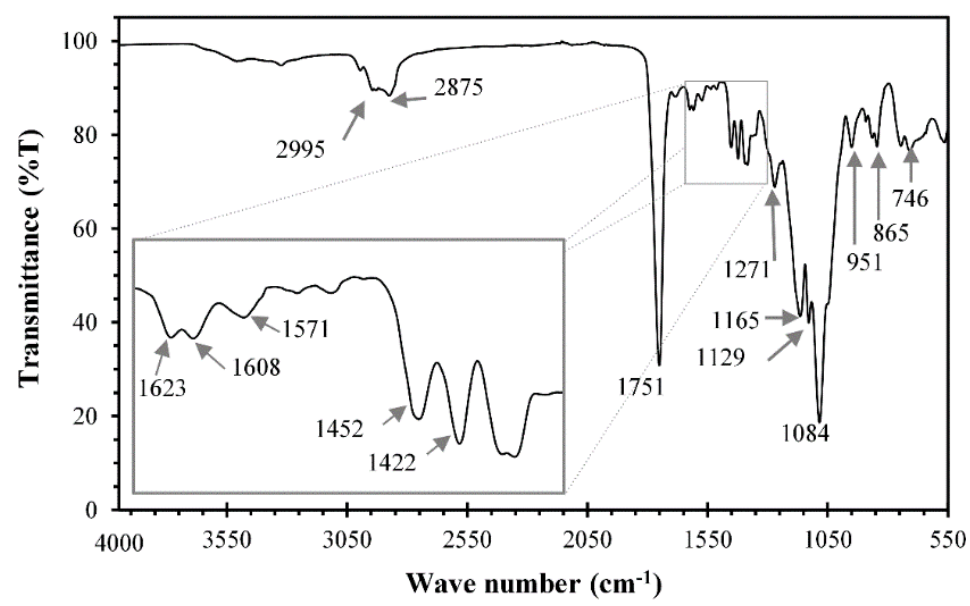

Figure 2. FT-IR absorption peaks of PLGA-PEG-FA polymer. Characteristic bands for aromatic ring present in folic acid at 1452 and $1608 \mathrm{~cm}^{-1}$ and PLGA-PEG-FA representative absorption peaks at 1623 and $1571 \mathrm{~cm}^{-1}$ due to the presence of -CONH- linkage.

\subsection{Nanoparticles Characterization}

Average values of particle size, polydispersity index (PDI), zeta-potential, DL and EE for different preparations of pDNA-NPs are shown in Table 1. Three different sets of experiments were performed by changing the initial amount of pVAX1-NH36 used in preparations, while the amount of polymers was maintained constant and related in the form of TDL. pDNA-NPs average diameter and average PDI could be observed in Figure 3A, where it is noted that the average diameters of three formulations are in the range of $170-230 \mathrm{~nm}$ and a correlation with TDL was also observed. In the range of study, diameter of pDNA-NPs increases with an increase in TDL.

Table 1. Characteristics of pDNA-NP as a function of theoretical pVAX1-NH36 loadings (pVAX1-NH36 TDL). Data represent mean \pm SD $(n=3)$.

\begin{tabular}{cccccc}
\hline pDNA-NP TDL (\%) & Diameter (nm) & PDI & Zeta Potential (mV) & DL (\%) & EE (\%) \\
\hline 0.2 & $177.2 \pm 18.2$ & $0.131 \pm 0.020$ & $-26.6 \pm 4.7$ & $0.0309 \pm 0.003$ & $15.437 \pm 1.56$ \\
0.5 & $202.1 \pm 12.1$ & $0.144 \pm 0.047$ & $-24.0 \pm 4.1$ & $0.0794 \pm 0.004$ & $15.881 \pm 0.74$ \\
0.8 & $229.3 \pm 20.0$ & $0.142 \pm 0.053$ & $-29.5 \pm 2.8$ & $0.1874 \pm 0.024$ & $23.429 \pm 2.96$ \\
\hline
\end{tabular}



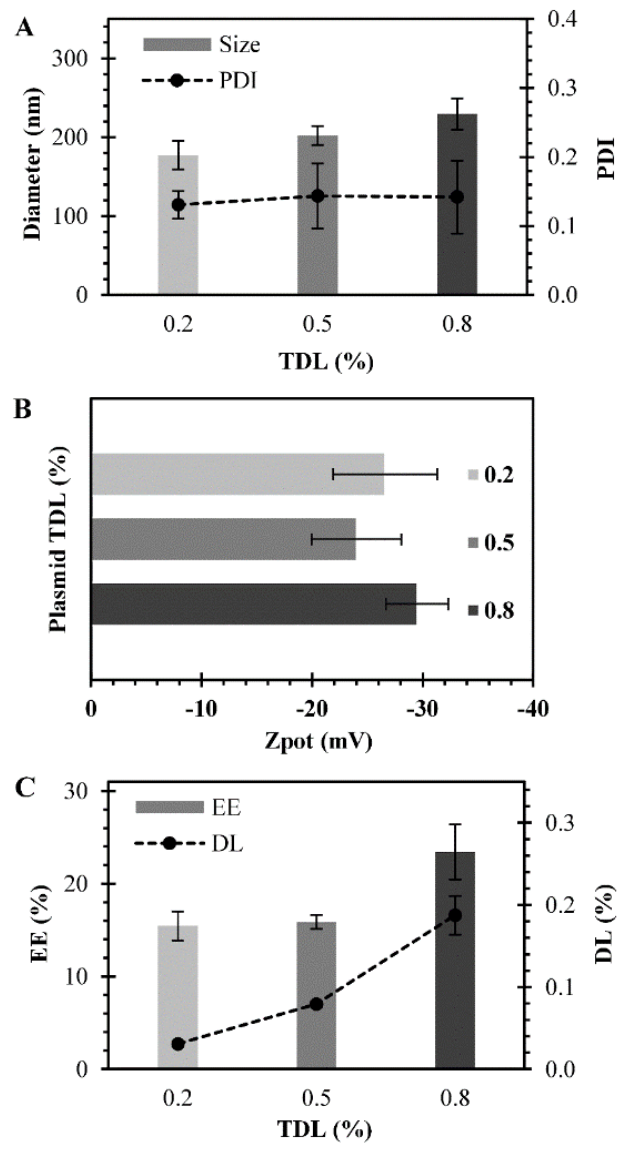

Figure 3. Characteristics of pDNA-NP prepared at different theoretical pVAX1-NH36 loadings (pVAX1-NH36 TDL). (A) Particle diameter and polydispersity index; (B) zeta potentials; (C) EE and DL for different pVAX1-NH36 with TDL of $0.2 \%(\square), 0.5 \%(\square)$ and $0.8 \%(\square)$ were evaluated. Data represent mean $\pm \mathrm{SD}(n=3)$

In previous works, Wang et al. performed plasmid encapsulation using PLGA with different molecular mass and obtained spherical microspheres in the range of 0.4 to $2.0 \mu \mathrm{m}$ [28]. Similarly, Gaspar et al. encapsulated plasmid for gene therapy using chitosan and obtained particles in the range of 157-198 nm [33]. Liang et al. and Esmaeili et al. have encapsulated different cancer chemotherapeutics using modified polymer PLGA-PEG-FA, obtaining particles in the range of 216-220 nm with polydispersity index of $0.225-0.113$ [12,13]. Yoo et al. also encapsulated an anticancer drug by conjugating the drug to PEG-FA, resulting in nano-aggregates with an average size of $200 \mathrm{~nm}$ [44]. Therefore, size range obtained in present research is appropriate for the type of particles being synthetized. Figure $3 \mathrm{~A}$ also exhibits average polydispersity index for pDNA-NPs ranges from 0.130 to 0.145 .

Surface charge is an important indication for stability of a colloidal nanoparticle system suspended in medium. Electrostatic repulsion among nanoparticles with the same type of surface charge provides extra stability [59]. Figure 3B show zeta potential obtained for diverse sets of experiments which vary between -26 to $-30 \mathrm{mV}$, indicating good stability. In the present work, variations in zeta potential with respect to different TDL do not present a significant statistical difference. Nanoparticle zeta potentials from previous works with similar polymer results in same kind of values $(-28.0 \mathrm{mV})$ are reported [13]. Other similar works, in which positively charged polymers such as polyethylenimine (PEI) $25 \mathrm{~K}$ or chitosan were being used, reported zeta potentials in the range of 20 to $50 \mathrm{mV}[11,33]$.

EE and DL are represented in Figure 3C. DL ranged between $0.03 \%$ and $0.19 \%$ as TDL increase. EE was $15.437 \%, 15.881 \%$ and $23.429 \%$ for corresponding TDL of $0.2 \%, 0.5 \%$ and $0.8 \%$. This trend could be explained if the double emulsification process employed, water/oil/water $(\mathrm{W} / \mathrm{O} / \mathrm{W})$ in the preparation of nanoparticles is considered. After the first emulsification, plasmid is mostly in the 
discontinuous phase (aqueous phase). In the second emulsification, some plasmid could migrate to the continuous water phase, decreasing the amount of plasmid that could be encapsulated. If the initial amount of plasmid used in the preparation is increased, then more plasmid could be retained in the discontinuous water phase, resulting in more plasmid physically entrapped into the nanoparticles, until some saturation point is reached. Comparing to other research reports, Wang et al. obtained an encapsulation efficiency of $53 \%$ in their experiments, but as previously noted, these experiments lead to larger particle size [28]. When encapsulating drugs such as cancer treatment medication, higher efficiencies can be achieved, as Esmaeili et al. reported an efficiency of $87.3 \%$ [12]. Yoo et al. encapsulated an anticancer drug conjugated with PEG-FA with a drug loading efficiency of $97.2 \%$ [60]. Gaspar et al. performed plasmid encapsulation in chitosan and obtained a particle loading capacity of $51.2 \%$ with an encapsulation efficiency of over $75 \%$ [33]. Benfer et al. reported encapsulating plasmid using PEG-PEI polyplexes with PLGA with encapsulation efficiencies of $26.4 \%-27.8 \%$ [11]. Shi et al. obtained a drug load in the range of $0.71 \%-0.77 \%$ which led to $88 \%-92 \%$ encapsulation efficiency when encapsulating a combination of cancer medication and plasmid DNA in porous PLGA nanoparticles and as of plasmid only, load was $0.036 \%-0.041 \%$ with plasmid encapsulation efficiency of $36 \%-40 \%$ [61]. It is noticeable that, higher efficiencies can be achieved during encapsulating medication in comparison with plasmid DNA, as negative charge of plasmid increases its encapsulation efficiency in PEI 25K [11,61]. However, PEI is non-degradable and molecular weight affects cytotoxicity and gene transfer activity; as high molecular weight PEI (PEI 25K) showed high transfection efficiency with significant cytotoxicity [62]. Although encapsulation efficiency obtained using PEI is larger, in this project, the polymers used in formulation of nanoparticles are degradable and/or do not present cytotoxicity issues. The morphology of pDNA-NPs is smooth and spherical as confirmed by SEM and AFM images as shown in Figure 4, with particles size in the range of 200-250 nm. Spherical morphology is congruent by other observations reported in literature for a similar system $[13,28,61]$.

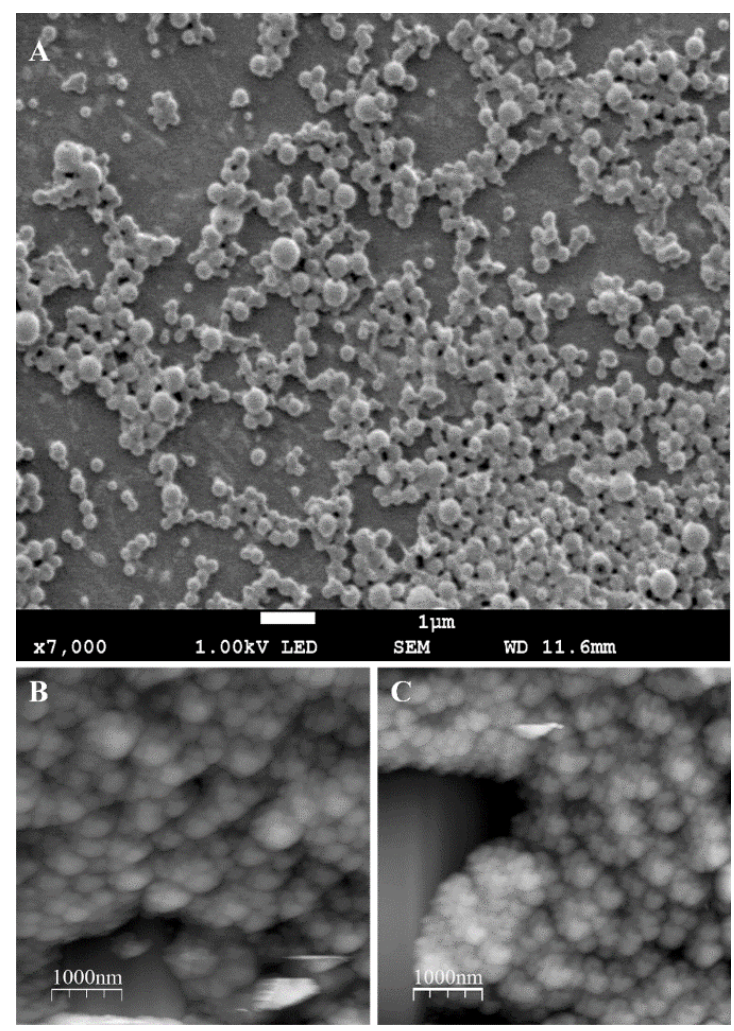

Figure 4. (A) Scanning electron microscopy (SEM) images of pDNA-NP with $0.8 \%$ theoretical pVAX1-NH36 loadings (TDL). Atomic force microscopy (AFM) images of pDNA-NP with (B) $0.2 \%$ TDL; and (C) $0.5 \%$ TDL. 


\subsection{Plasmid Release Analysis}

In vitro release studies of pVAX1-NH36 from pDNA-NPs were performed with three different TDL used in preparations and are presented in Figure 5. During the first days of the study, initial burst rate was proportionally related to DL of pVAX1-NH36. Amounts of pVAX1-NH36 released in these initial burst stages were around $0.44,1.88$ and $10.39 \mu \mathrm{g}$, corresponding to $13 \%, 22 \%$ and $55 \%$ for TDL of $0.2 \%, 0.5 \%$ and $0.8 \%$ respectively. Since pVAX1-NH36 is hydrophilic in nature, we hypothesize that most of the plasmid was encapsulated close to the surface of nanoparticles, which could explain the initial high rate of release by rapid dissolution of pVAX1-NH36 in release media. After the initial burst stage, a slow release was observed for three TDL evaluated until a plateau was reached, indicating that all pVAX1-NH36 was released from pDNA-NPs. This retardation in release after initial burst could be explained by physical entrapment of pVAX1-NH36 and low rate of water penetration into nanoparticle. Plasmid is then slowly released by degradation-relaxation of NPs. In addition, PLGA NPs hydrolysis promotes water permeation and transport of plasmid into media. Similar results have been presented by Shi et al. with an initial burst of $40 \%$ within the first four hours of the study, followed by a slower release rate period of seven days with a cumulative release of $60 \%$ after day 1 and $70 \%$ after day 3 [61]. The difference in release times could be attributed to porosity of particles. Likewise, Liang et al. observed the same profile behavior with release of a drug from PLGA-PEG-FA nanoparticles with an initial burst release of $20 \%$ in the first two hours, followed by a cumulative release of $40 \%$ by the next $12 \mathrm{~h}$, and then a slow linear release until it reached $80 \%$ at day 7 [13].
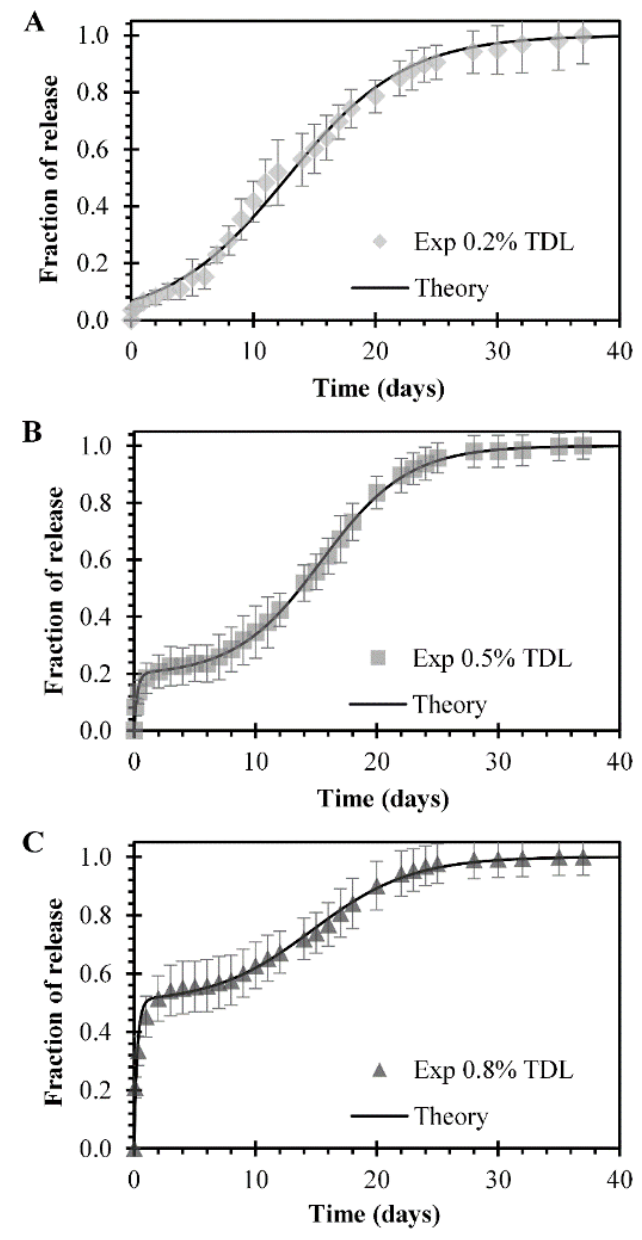

Figure 5. In vitro pVAX1-NH36 release profiles from pDNA-NP for theoretical pVAX1-NH36 loadings $(\mathrm{TDL})$ of $0.2 \%(\diamond), 0.5 \%(\square)$ and $0.8 \%(\Delta)$. Data represent mean $(n=3)$. 
Experimental pVAX1-NH36 release data was analyzed by a coupled model that incorporates initial burst as well as NPs degradation-relaxation (Equation (3)). In general, an excellent fit of the proposed model with experimental profiles was obtained. Figure $5 \mathrm{~A}-\mathrm{C}$ shows the release profiles of pVAX1-NH36 for TDL of $0.2 \%, 0.5 \%$ and $0.8 \%$, where experimental data is represented by diamonds, squares and triangles in figures, respectively. For all cases, fitting of the model to experimental release was denoted by a solid line. Model parameters obtained by fitting are presented in Table 2 . Burst constant decreases from 4.4513 to 3.6538 days $^{-1}$ when TDL increases from $0.2 \%$ to $0.5 \%$ and is maintained in the same range even though TDL increases up to $0.8 \%$. Fraction of burst release increases proportionally with TDL, from $1.0587 \times 10^{-8}$ to 0.1914 and finally to 0.4977 for TDL of $0.2 \%, 0.5 \%$ and $0.8 \%$ respectively. Degradation-relaxation constant increases from 0.2060 to 0.2792 days $^{-1}$ for $0.2 \%$ and $0.5 \% \mathrm{TDL}$, followed by a decrease to 0.2439 days $^{-1}$ for $0.8 \%$ TDL. The time to achieve $50 \%$ of release was maintained in the range of 12 to 15 days for all cases. As expected, fraction of NPs relaxation release behaves in the opposite way to fraction of burst release, decreasing from 0.9999 to 0.8086 to 0.5023 for TDL of $0.2 \%, 0.5 \%$ and $0.8 \%$ respectively. The proposed model of release, presented in Equation (3), simultaneously considers the stages of initial burst and NPs degradation-relaxation. Therefore, depending on experimental data, the fraction values of burst $\left(\theta_{b}\right)$ or NP relaxation $\left(\theta_{r}\right)$ reflected contributions of each mechanism over the complete release profile. Values obtained with the model are within the same range as others reported in literature for release of small molecules (ketoprofen, indomethacin, and coumarin-6) and macromolecules (human serum albumin, ovalbumin) [40]. Finally, resulted coefficients of determination for all fittings were greater than 0.99 and thus ensure the validity of the present model.

Table 2. Parameters of plasmid pVAX1-NH36 release from pDNA-NP. The parameters were determined and used in the mathematical development of the release model.

\begin{tabular}{cccccc}
\hline \multirow{2}{*}{ Parameters } & Description & Unit & \multicolumn{3}{c}{ TDL (\%) } \\
\cline { 3 - 6 } & & & $\mathbf{0 . 2}$ & $\mathbf{0 . 5}$ & $\mathbf{0 . 8}$ \\
\hline$k_{b}$ & Burst constant & days $^{-1}$ & 4.4513 & 3.6538 & 3.6722 \\
$\theta_{b}$ & Fraction of burst release & - & $1.059 \times 10^{-8}$ & 0.1914 & 0.4977 \\
$k_{r}$ & Degradation-relaxation constant & days $^{-1}$ & 0.2060 & 0.2792 & 0.2439 \\
$t_{\max }$ & Time to achieve 50\% of release & days & 12.7481 & 15.4318 & 14.6954 \\
$\theta_{r}$ & Fraction of NP relaxation release & - & 0.9999 & 0.8086 & 0.5023 \\
$R^{2}$ & Coefficient of determination & - & 0.9961 & 0.9996 & 0.9969 \\
\hline
\end{tabular}

\subsection{Plasmid Purity and Integrity}

Figure 6 presents a gel electrophoresis image obtained from different samples of pVAX1-NH36 at different stages of the nanoparticle preparation process. These samples were taken to verify initial purity of pVAX1-NH36, and also to evaluate its integrity towards the nanoparticle preparation process. Future studies should include transfect $E$. coli with recovered pDNA and produce kanamycin resistance, thus demonstrating that released pDNA remained functional. Figure 6 presents four channels, where the first one shows the size markers for plasmid over the range of 2972-12,138 bp. Channel two presents initial pVAX1-NH36 solution used to formulate pDNA-NPs. This solution contains pVAX1-NH36 in its supercoiled form (band of $4000 \mathrm{bp}$ ) and two relaxed isoforms. Channel three and four display samples obtained after pVAX1-NH36 was released from pDNA-NPs for two different batches prepared with $0.5 \%$ and $0.8 \%$ TDL respectively. Gel image showed single bands representing supercoiled and two relaxed forms of pVAX1-NH36 plasmid consistent with initial plasmid solution bands. Difference in brightness is due to difference of pVAX1-NH36 concentration in each sample. In accordance with electrophoresis gel, plasmid quality over encapsulation experiments was constant without any noticeable damage. Studies of integrity of pure pVAX1-NH36 in aqueous solution during the use of sonication at different potencies were performed and no plasmid damage was observed by using the technique of electrophoresis in agarose gel (data not shown in this work). Wang et al. thoroughly 
tested structural and functional integrity over sonication experiments and their results suggested that polymer (PLGA) protects plasmid DNA from being directly affected by sheering due to high energy of sonication [28]. Shi et al. verified plasmid integrity after performing electrophoresis gels confirming that plasmid was amenable to gene transfection [61].

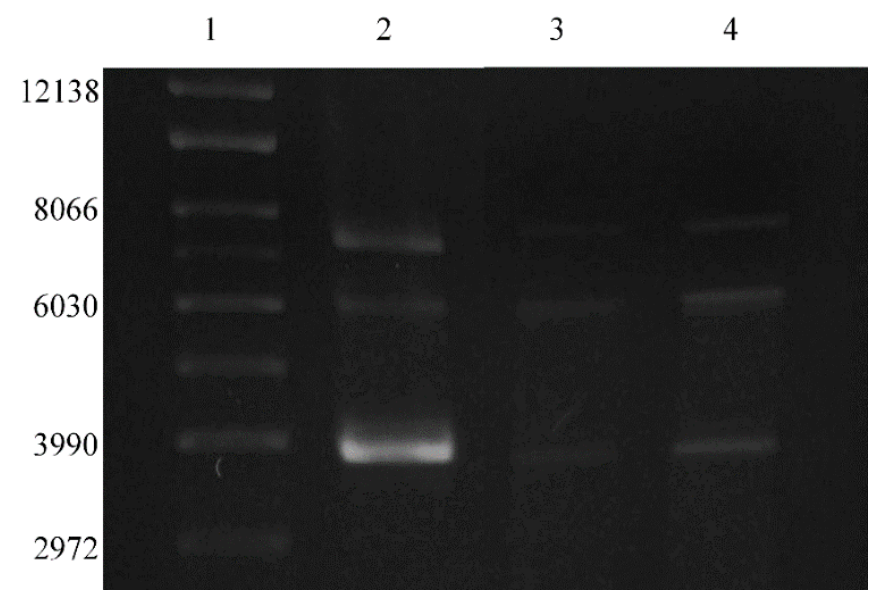

Figure 6. Agarose gel electrophoresis for pVAX1-NH36 before and after nanoparticle preparation. (1) Size marker for supercoiled plasmid form over the range 2972-12,138 bp; (2) Initial pVAX1-NH36 solution used to formulate the pDNA-NP; (3) Sample acquired from $0.5 \%$ theoretical pVAX1-NH36 loadings (TDL) solution after pDNA-NP purification; (4) Sample obtained from 0.8\% TDL solution after pDNA-NP purification.

\section{Conclusions}

PLGA-PEG-FA copolymer was successfully constructed by two conjugation steps and was characterized by FITR. Plasmid pVAX1-NH36 was propagated and efficiently encapsulated into modified polymer PLGA-PEG-FA nanoparticles. Size, polydispersity index, zeta potential, encapsulation efficiency and plasmid load of nanoparticles were determined. These results suggest that with an increase in TDL, diameter of pDNA-NPs, encapsulation efficiency and drug loading increase. However, zeta potential and polydispersity index did not present a significant statistical difference, suggesting stability of the particles. Experimental pVAX1-NH36 release profile shows a multiphase behavior for all three TDL investigated. A mathematical analysis was introduced to describe experimental release profile by considering simultaneous contributions of initial burst and degradation-relaxation of NPs. Fitting of the release model against experimental data presents an excellent correlation. This mathematical analysis could be used to describe and predict the release of plasmid DNA from biodegradable NPs. In addition, pVAX1-NH36 quality over encapsulation experiments was constant without any noticeable plasmid damage.

Acknowledgments: Financial assistance from the National Council of Science and Technology of Mexico (CONACYT) is genuinely acknowledged with projects I0007-2013-02 No. 206489 and INFR-2014-01 No. 226208. Reynaldo Esquivel thanks CONACYT for projects PDCPN201401-247326. We thank Luis Angel Medina Suárez group for their assistance in the lyophilization of NP, Josué Juárez Onofre for his support with the AFM micrographs, and also Roberto Carrillo for his help with the SEM acquisition. Cindy Alejandra Gutiérrez-Valenzuela thanks CONACYT for the Ph.D. scholarship.

Author Contributions: Cindy Alejandra Gutiérrez-Valenzuela performed all experiments and contributed in the manuscript writing. Patricia Guerrero-Germán, Armando Tejeda-Mansir, and Roberto Guzmán-Z contributed with reagents/materials/analysis tools and discussion for all experiments. Reynaldo Esquivel contributed in the manuscript discussion and writing. Armando Lucero-Acuña conceived and designed the experiments and contributed in the manuscript writing.

Conflicts of Interest: The authors declare no conflict of interest. 


\section{References}

1. Santini, J.T. Introduction to drug delivery technology. Chem. Eng. Process. 2013, 109, 19-23.

2. Shi, J.; Votruba, A.R.; Farokhzad, O.C.; Langer, R. Nanotechnology in drug delivery and tissue engineering: From discovery to applications. Nano Lett. 2010, 10, 3223-3230. [CrossRef] [PubMed]

3. Peer, D.; Karp, J.M.; Hong, S.; Farokhzad, O.C.; Margalit, R.; Langer, R. Nanocarriers as an emerging platform for cancer therapy. Nat. Nanotechnol. 2007, 2, 751-760. [CrossRef] [PubMed]

4. Danhier, F.; Ansorena, E.; Silva, J.M.; Coco, R.; Le Breton, A.; Préat, V. PLGA-based nanoparticles: An overview of biomedical applications. J. Control. Release 2012, 161, 505-522. [CrossRef] [PubMed]

5. Makadia, H.K.; Siegel, S.J. Poly lactic-co-glycolic acid (PLGA) as biodegradable controlled drug delivery carrier. Polymers 2011, 3, 1377-1397. [CrossRef] [PubMed]

6. Jain, R.A. The manufacturing techniques of various drug loaded biodegradable poly(lactide-co-glycolide) (PLGA) devices. Biomaterials 2000, 21, 2475-2490. [CrossRef]

7. Kumari, A.; Yadav, S.K.; Yadav, S.C. Biodegradable polymeric nanoparticles based drug delivery systems. Colloids Surf. B Biointerfaces 2010, 75, 1-18. [CrossRef] [PubMed]

8. Peracchia, M.T.; Gref, R.; Minamitake, Y.; Domb, A.; Lotan, N.; Langer, R. PEG-coated nanospheres from amphiphilic diblock and multiblock copolymers: Investigation of their drug encapsulation and release characteristics. J. Control. Release 1997, 46, 223-231. [CrossRef]

9. Paun, I.A.; Moldovan, A.; Luculescu, C.R.; Staicu, A.; Dinescu, M. MAPLE deposition of PLGA:PEG films for controlled drug delivery: Influence of PEG molecular weight. Appl. Surf. Sci. 2012, 258, 9302-9308. [CrossRef]

10. Parveen, S.; Sahoo, S.K. Polymeric nanoparticles for cancer therapy. J. Drug Target. 2008, 16, 108-123. [CrossRef] [PubMed]

11. Benfer, M.; Reul, R.; Betz, T.; Kissel, T. Folic acid-decorated nanocomposites prepared by a simple solvent displacement method. Macromol. Biosci. 2012, 12, 438-445. [CrossRef] [PubMed]

12. Esmaeili, F.; Ghahremani, M.H.; Ostad, S.N.; Atyabi, F.; Seyedabadi, M.; Malekshahi, M.R.; Amini, M.; Dinarvand, R. Folate-receptor-targeted delivery of docetaxel nanoparticles prepared by PLGA-PEG-folate conjugate. J. Drug Target. 2008, 16, 415-423. [CrossRef] [PubMed]

13. Liang, C.; Yang, Y.; Ling, Y.; Huang, Y.; Li, T.; Li, X. Improved therapeutic effect of folate-decorated PLGA-PEG nanoparticles for endometrial carcinoma. Bioorg. Med. Chem. 2011, 19, 4057-4066. [CrossRef] [PubMed]

14. Yang, J.; Lee, C.-H.; Park, J.; Seo, S.; Lim, E.-K.; Song, Y.J.;Suh, J.-S.; Yoon, H.-G.; Huh, Y.-M.; Haam, S. Antibody conjugated magnetic PLGA nanoparticles for diagnosis and treatment of breast cancer. J. Mater. Chem. 2007, 17, 2695-2699. [CrossRef]

15. Grazia Cascone, M.; Zhu, Z.; Borselli, F.; Lazzeri, L. Poly(vinyl alcohol) hydrogels as hydrophilic matrices for the release of lipophilic drugs loaded in PLGA nanoparticles. J. Mater. Sci. Mater. Med. 2002, 13, 29-32. [CrossRef] [PubMed]

16. Murakami, H.; Kobayashi, M.; Takeuchi, H.; Kawashima, Y. Further application of a modified spontaneous emulsification solvent diffusion method to various types of PLGA and PLA polymers for preparation of nanoparticles. Powder Technol. 2000, 107, 137-143. [CrossRef]

17. Sahana, D.K.; Mittal, G.; Bhardwaj, V.; Kumar, M.N.V.R. PLGA nanoparticles for oral delivery of hydrophobic drugs: Influence of organic solvent on nanoparticle formation and release behavior in vitro and in vivo using estradiol as a model drug. J. Pharm. Sci. 2008, 97, 1530-1542. [CrossRef] [PubMed]

18. Dhar, S.; Gu, F.X.; Langer, R.; Farokhzad, O.C.; Lippard, S.J. Targeted delivery of cisplatin to prostate cancer cells by aptamer functionalized Pt(IV) prodrug-PLGA-PEG nanoparticles. Proc. Natl. Acad. Sci. USA 2008, 105, 17356-17361. [CrossRef] [PubMed]

19. Chorny, M.; Fishbein, I.; Danenberg, H.D.; Golomb, G. Lipophilic drug loaded nanospheres prepared by nanoprecipitation: Effect of formulation variables on size, drug recovery and release kinetics. J. Control. Release 2002, 83, 389-400. [CrossRef]

20. Iborra, S.; Soto, M.; Carrión, J.; Alonso, C.; Requena, J.M. Vaccination with a plasmid DNA cocktail encoding the nucleosomal histones of Leishmania confers protection against murine cutaneous leishmaniosis. Vaccine 2004, 22, 3865-3876. [CrossRef] [PubMed]

21. Jain, K.; Jain, N.K. Vaccines for visceral leishmaniasis: A review. J. Immunol. Methods 2015, 422, 1-12. [CrossRef] [PubMed] 
22. Chalé-balboa, W.G.; Mut-Martin, M.; Ramirez-Sierra, M.J.; Garcia-Miss, M.R.; Dumonteil, E. A combination DNA vaccine encoding nucleoside hydrolase 36 and glycoproteine 63 protects female but not male hamsters against Leishmania mexicana. Parasite 2009, 16, 227-230. [CrossRef] [PubMed]

23. Gamboa-León, R.; Paraguai de Souza, E.; Borja-Cabrera, G.P.; Santos, F.N.; Myashiro, L.M.; Pinheiro, R.O.; Dumonteil, E.; Palatnik-de-Sousa, C.B. Immunotherapy against visceral leishmaniasis with the nucleoside hydrolase-DNA vaccine of Leishmania donovani. Vaccine 2006, 24, 4863-4873. [CrossRef] [PubMed]

24. Dumonteil, E. DNA vaccines induce partial protection against Leishmania mexicana. Vaccine 2003, 21, 2161-2168. [CrossRef]

25. Ismail, R.; Allaudin, Z.N.; Lila, M.-A.M. Scaling-up recombinant plasmid DNA for clinical trial: Current concern, solution and status. Vaccine 2012, 30, 5914-5920. [CrossRef] [PubMed]

26. Nagill, R.; Kaur, S. Vaccine candidates for leishmaniasis: A review. Int. Immunopharmacol. 2011, 11, $1464-1488$. [CrossRef] [PubMed]

27. Palatnik-de-Sousa, C.B. Vaccines for leishmaniasis in the fore coming 25 years. Vaccine 2008, 26, $1709-1724$. [CrossRef] [PubMed]

28. Wang, D.; Robinson, D.R.; Kwon, G.S.; Samuel, J. Encapsulation of plasmid DNA in biodegradable poly(D,L-lactic-co-glycolic acid) microspheres as a novel approach for immunogene delivery. J. Control. Release 1999, 57, 9-18. [CrossRef]

29. Basto, P.A. Development of Polymeric Nanoparticle Vaccines for Immunostimulation. Ph.D. Thesis, Massachusetts Institute of Technology, Cambridge, MA, USA, 2013.

30. Kunugi, S.; Yamaoka, T. Polymers in Nanomedicine; Kunugi, S., Yamaoka, T., Eds.; Advances in Polymer Science; Springer: Berlin/Heidelberg, Germany, 2012; Volume 247.

31. Schaffert, D.; Wagner, E. Gene therapy progress and prospects: Synthetic polymer-based systems. Gene Ther. 2008, 15, 1131-1138. [CrossRef] [PubMed]

32. Jayakumar, R.; Chennazhi, K.P.; Muzzarelli, R.A.A.; Tamura, H.; Nair, S.V.; Selvamurugan, N. Chitosan conjugated DNA nanoparticles in gene therapy. Carbohydr. Polym. 2010, 79, 1-8. [CrossRef]

33. Gaspar, V.M.; Correia, I.J.; Sousa, A.; Silva, F.; Paquete, C.M.; Queiroz, J.A.; Sousa, F. Nanoparticle mediated delivery of pure P53 supercoiled plasmid DNA for gene therapy. J. Control. Release 2011, 156, $212-222$. [CrossRef] [PubMed]

34. Saljoughian, N.; Zahedifard, F.; Doroud, D.; Doustdari, F.; Vasei, M.; Papadopoulou, B.; Rafati, S. Cationic solid-lipid nanoparticles are as efficient as electroporation in DNA vaccination against visceral leishmaniasis in mice. Parasite Immunol. 2013, 35, 397-408. [CrossRef] [PubMed]

35. Perez, C.; Sanchez, A.; Putnam, D.; Ting, D.; Langer, R.; Alonso, M. Poly(lactic acid)-poly(ethylene glycol) nanoparticles as new carriers for the delivery of plasmid DNA. J. Control. Release 2001, 75, 211-224. [CrossRef]

36. Zhao, K.; Zhang, Y.; Zhang, X.; Shi, C.; Wang, X.; Wang, X.; Jin, Z.; Cui, S. Chitosan-coated poly(lactic-co-glycolic) acid nanoparticles as an efficient delivery system for Newcastle disease virus DNA vaccine. Int. J. Nanomed. 2014, 9, 4609-4619. [CrossRef] [PubMed]

37. Megeed, Z.; Cappello, J.; Ghandehari, H. Controlled Release of Plasmid DNA from a Genetically Engineered Silk-Elastinlike Hydrogel. Pharm. Res. 2002, 19, 954-959. [CrossRef] [PubMed]

38. Fredenberg, S.; Wahlgren, M.; Reslow, M.; Axelsson, A. The mechanisms of drug release in poly(lactic-co-glycolic acid)-based drug delivery systems-A review. Int. J. Pharm. 2011, 415, 34-52. [CrossRef] [PubMed]

39. Lao, L.L.; Peppas, N.A.; Boey, F.Y.C.; Venkatraman, S.S. Modeling of drug release from bulk-degrading polymers. Int. J. Pharm. 2011, 418, 28-41. [CrossRef] [PubMed]

40. Corrigan, O.I.; Li, X. Quantifying drug release from PLGA nanoparticulates. Eur. J. Pharm. Sci. 2009, 37, 477-485. [CrossRef] [PubMed]

41. Fitzgerald, J.F.; Corrigan, O.I. Mechanisms governing drug release from poly- $\alpha$-hydroxy aliphatic esters. Diltiazem base release from poly-lactide-co-glycolide delivery systems. In Polymeric Delivery Systems; American Chemical Society: Washington, DC, USA, 1993; pp. 311-326.

42. Prout, E.G.; Tompkins, F.C. The thermal decomposition of potassium permanganate. Trans. Faraday Soc. 1944, 40, 488-498. [CrossRef]

43. Nie, Y.; Zhang, Z.; Li, L.; Luo, K.; Ding, H.; Gu, Z. Synthesis, characterization and transfection of a novel folate-targeted multipolymeric nanoparticles for gene delivery. J. Mater. Sci. Mater. Med. 2009, 20, 1849-1857. [CrossRef] [PubMed] 
44. Yoo, H.S.; Park, T.G. Folate-receptor-targeted delivery of doxorubicin nano-aggregates stabilized by doxorubicin-PEG-folate conjugate. J. Control. Release 2004, 100, 247-256. [CrossRef] [PubMed]

45. Boddu, S.H.S.; Vaishya, R. Preparation and Characterization of Folate Conjugated Nanoparticles of Doxorubicin using PLGA-PEG-FOL Polymer. Med. Chem. 2012, 2, 068-075. [CrossRef]

46. Hans, M.; Lowman, A. Biodegradable nanoparticles for drug delivery and targeting. Curr. Opin. Solid State Mater. Sci. 2002, 6, 319-327. [CrossRef]

47. Lucero-Acuna, J.A. Synthesis and Characterization of Polymeric Nanoparticle Structures for Control Drug Delivery in cancer Therapies and Temperature Effects on Drug Release. Ph.D. Thesis, The University of Arizona, Tucson, AZ, USA, 2013.

48. Horcas, I.; Fernández, R.; Gómez-Rodríguez, J.M.; Colchero, J.; Gómez-Herrero, J.; Baro, A.M. WSXM: A software for scanning probe microscopy and a tool for nanotechnology. Rev. Sci. Instrum. 2007, 78, 013705. [CrossRef] [PubMed]

49. Menon, J.U.; Ravikumar, P.; Pise, A.; Gyawali, D.; Hsia, C.C.W.; Nguyen, K.T. Polymeric nanoparticles for pulmonary protein and DNA delivery. Acta Biomater. 2014, 10, 2643-2652. [CrossRef] [PubMed]

50. Son, S.; Kim, W.J. Biodegradable nanoparticles modified by branched polyethylenimine for plasmid DNA delivery. Biomaterials 2010, 31, 133-143. [CrossRef] [PubMed]

51. Diogo, M.M.; Queiroz, J.A.; Prazeres, D.M.F. Assessment of purity and quantification of plasmid DNA in process solutions using high-performance hydrophobic interaction chromatography. J. Chromatogr. A 2003, 998, 109-117. [CrossRef]

52. Soppimath, K.S.; Aminabhavi, T.M.; Kulkarni, A.R.; Rudzinski, W.E. Biodegradable polymeric nanoparticles as drug delivery devices. J. Control. Release 2001, 70, 1-20. [CrossRef]

53. Batycky, R.P.; Hanes, J.; Langer, R.; Edwards, D.A. A theoretical model of erosion and macromolecular drug release from biodegrading microspheres. J. Pharm. Sci. 1997, 86, 1464-1477. [CrossRef] [PubMed]

54. Lucero-Acuña, A.; Guzmán, R. Nanoparticle encapsulation and controlled release of a hydrophobic kinase inhibitor: Three stage mathematical modeling and parametric analysis. Int. J. Pharm. 2015, 494, $249-257$. [CrossRef] [PubMed]

55. Dunne, M.; Corrigan, I.; Ramtoola, Z. Influence of particle size and dissolution conditions on the degradation properties of polylactide-co-glycolide particles. Biomaterials 2000, 21, 1659-1668. [CrossRef]

56. Lao, L.L.; Venkatraman, S.S.; Peppas, N.A. A novel model and experimental analysis of hydrophilic and hydrophobic agent release from biodegradable polymers. J. Biomed. Mater. Res. A 2009, 90, 1054-1065. [CrossRef] [PubMed]

57. Choy, J.-H.; Jung, J.-S.; Oh, J.-M.; Park, M.; Jeong, J.; Kang, Y.-K.; Han, O.-J. Layered double hydroxide as an efficient drug reservoir for folate derivatives. Biomaterials 2004, 25, 3059-3064. [CrossRef] [PubMed]

58. Yang, S.-J.; Lin, F.-H.; Tsai, K.-C.; Wei, M.-F.; Tsai, H.-M.; Wong, J.-M.; Shieh, M.-J. Folic acid-conjugated chitosan nanoparticles enhanced protoporphyrin IX accumulation in colorectal cancer cells. Bioconj. Chem. 2010, 21, 679-689. [CrossRef] [PubMed]

59. Liu, Y.; Li, K.; Pan, J.; Liu, B.; Feng, S.-S. Folic acid conjugated nanoparticles of mixed lipid monolayer shell and biodegradable polymer core for targeted delivery of Docetaxel. Biomaterials 2010, 31, 330-338. [CrossRef] [PubMed]

60. Yoo, H.S.; Park, T.G. Folate receptor targeted biodegradable polymeric doxorubicin micelles. J. Control. Release 2004, 96, 273-283. [CrossRef] [PubMed]

61. Shi, X.; Li, C.; Gao, S.; Zhang, L.; Han, H.; Zhang, J.; Shi, W.; Li, Q. Combination of doxorubicin-based chemotherapy and polyethylenimine/p53 gene therapy for the treatment of lung cancer using porous PLGA microparticles. Colloids Surf. B Biointerfaces 2014, 122, 498-504. [CrossRef] [PubMed]

62. Wen, Y.; Pan, S.; Luo, X.; Zhang, X.; Zhang, W.; Feng, M. A biodegradable low molecular weight polyethylenimine derivative as low toxicity and efficient gene vector. Bioconj. Chem. 2009, 20, 322-332. [CrossRef] [PubMed]

(C) 2016 by the authors; licensee MDPI, Basel, Switzerland. This article is an open access article distributed under the terms and conditions of the Creative Commons Attribution (CC-BY) license (http://creativecommons.org/licenses/by/4.0/). 